Revista de

Contabilidade e

Organizações

www.rco.usp.br
DOI: http://dx.doi.org/10.11606/rco.v10i26.107117
Journal of

Accounting and

Organizations www.rco.usp.br

\title{
Nota fiscal de serviços eletrônica: uma análise dos impactos na arrecadação em municípios brasileiros
}

\author{
Humberto de Angeli Neto ${ }^{\text {a }}$ Antonio Lopo Martinez ${ }^{\mathrm{a}}$
}

${ }^{a}$ FUCAPE Business School

\section{Informações do Artigo}

Histórico do Artigo

Recebido: 05 de novembro de 2015

Aceito: 24 de abril de 2016

Palavras-chave:

Governos eletrônicos.

Nota fiscal de serviços eletrônica.

SPED.

\begin{abstract}
Resumo
Este artigo analisou a evolução dos sistemas informacionais desenvolvidos pelos governos (em especial as notas fiscais eletrônicas) como uma forma de possibilitar uma maior arrecadação para as administrações tributárias. Nesta pesquisa procurouse investigar a relação entre os aumentos de arrecadação no imposto de serviços sobre qualquer natureza (ISSQN) per capita e os efeitos da implantação do sistema de notas fiscais de serviços eletrônicas (NFS-e) nos municípios brasileiros mais populosos. Verificou-se que não há evidência de um aumento da arrecadação de impostos sobre qualquer natureza (ISSQN) per capita após a implantação das notas fiscais de serviços eletrônicas. Esses resultados foram semelhantes aos apresentados nos estudos de Mattos et al (2013) que analisou o impacto do Programa Nota Fiscal Paulista sobre a arrecadação do Estado de São Paulo. Apesar de não ser objeto da pesquisa, é inegável a externalidade positiva em termos de eficiência para a Administração Tributária, assim como a redução dos custos de transação, que a utilização da NFS-e promove, trazendo benefícios para toda a sociedade.
\end{abstract}

Copyright (C) 2016 FEA-RP/USP. Todos os direitos reservados

\section{INTRODUÇÃO}

No cenário internacional, Winn e Zhang (2013) mostram que de uma forma geral todas as grandes economias tiveram uma preocupação em padronizar seus sistemas de impostos e disponibilizar um sistema eletrônico de emissão de notas fiscais. Para Quest e Sharma (2009), os impostos estão entrando na era da tecnologia. Sistemas de informação já são amplamente utilizados na gestão comercial e de produção, bem como agora passam a gestão de riscos tributários e serão integrados on-line com a administração tributária.

Winn e Zhang (2013) adicionam que o uso da nota fiscal eletrônica é uma tendência dos governos pelo incremento do poder de monitoramento do estado ao pagamento de impostos e aumento da competitividade global nos locais onde é implantado.

$\mathrm{Na}$ Europa, as autoridades fiscais identificaram vários caminhos para o aumento da eficiência nos negócios europeus mediante o uso de um sistema de notas fiscais eletrônicas em larga escala. Dentre os benefícios listados estão: i) o aumento da competividade pelo incremento da produtividade e satisfação do cliente; ii) alcance de substanciais reduções de custos através da redução do trabalho manual, material e custos de transporte, iii) aumento do fluxo de caixa pela redução de perdas de créditos face o aumento da automação no processamento de pagamentos (WINN e ZHANG, 2013).

Apesar da Europa ter iniciado seus trabalhos de criação de um sistema de negócios informatizados com a definição de regras gerais, viu-se que o ganho de valor seria maior com uma regulação das tecnologias a serem adotadas. Em junho de 2010 a comunidade europeia resolveu recomendar tanto para o setor privado como o público a adoção de um modelo padrão de nota fiscal e um modelo de dados. Esse modelo de centralização foi semelhante ao caminho adotado pelos Estados Unidos e resultou na plataforma ISO 20022 (WINN e ZHANG, 2013).

Autor Correspondente: Tel (27) 4009-4444

E-mail: humberto.angeli@gmaill.com (H. A. Neto); antoniolopo@terra.com.br (A. L. Martinez)

FUCAPE - Av. Fernando Ferrari, 1358 - Boa Visra, Vitória - ES, 29075-505, Brasil. 
Ainda conforme Winn e Zhang (2013), a China também teve seu projeto de informatização de sua nota fiscal. O governo central lançou "Golden Projects" para promover o desenvolvimento de sua infraestrutura e modernizar a entrega de serviços do governo em projetos nos anos 80 e 90 (1986-1990 e 1991-1996). Um desses projetos foi o Golden Tax Project, focado no uso da tecnologia da informação para aumentar a aplicação das leis tributárias.

Por sua vez, no Brasil, conforme Portal da Nota Fiscal Eletrônica Brasileiro, as primeiras iniciativas de automatização da arrecadação começaram a surgir com o convênio ICMS de 156/94 que regulamentou e estabeleceu a obrigatoriedade do uso dos sistemas de automação comercial para determinados tipos de empresa na esfera federal. Os governos estaduais e municipais atentos a essa tendência também buscaram juntar-se a todas essas iniciativas.

Em 2004 ocorreu o $1^{\circ}$ Encontro Nacional de Administradores Tributários - ENAT, reuniu administradores em âmbito federal, estaduais, Distrito Federal e dos municípios de capitais (BEZERRA, 2010). No ENAT buscouse uma solução conjunta das três esferas do governo para promoverem uma maior integração administrativa, padronização e melhor qualidade das informações visando a diminuição dos custos, carga de trabalho no atendimento e um aumento da arrecadação (DUARTE, 2009). Nesse encontro foram aprovados dois protocolos de cooperação tecnológica em áreas do cadastramento (Projeto do Cadastro Sincronizado) e da Nota Fiscal Eletrônica (BEZERRA, 2010).

Azevedo e Mariano (2014) informam que em abril de 2005 houve o II ENAT que focou a discussão e a elaboração de projetos sobre o Cadastro Sincronizado Nacional e o Sistema Público de Escrituração Digital (SPED) onde a implantação da Nota Fiscal Eletrônica seria um de seus principais objetivos.

Diante desse contexto, torna-se relevante perquirir se houve ganho de arrecadação nas localidades onde foram implantadas as notas fiscais eletrônicas. Se a literatura indica que o controle aumenta, será que a nota fiscal eletrônica propiciaria um aumento de arrecadação efetivo, ou apenas ela supre a necessidade das administrações se adaptarem a economias crescentes e cada vez mais complexas que exigem da administração tributária medidas mais eficientes para a gestão e controle tributário. Isto posto, este trabalho tem como problemática de pesquisa a seguinte questão: A implantação da nota fiscal eletrônica gerou aumento de arrecadação nas localidades onde foi implantado?

No item seguinte será apresentado o referencial teórico em torno da problemática. Seguido por um item voltado a discussão dos aspectos metodológicos expondo os procedimentos adotados para confrontar o problema. Na parte seguinte se expõe os resultados da pesquisa e são feitas as análises em torno dos achados. Finalmente é apresentada a conclusão da pesquisa com as descobertas mais relevantes.

\section{REFERENCIAL TEÓRICO}

\section{1 A necessidade de um Sistema tributário eficiente}

Harberger (1959) foi um dos primeiros a escrever um estudo correlacionando o efeito da tributação na economia. A evolução desses estudos ficou conhecido como o "Triângulo de Harberger", que refere-se ao peso morto causado pelos impostos nos mercados devido a intervenção do governo. Essa forma de um triângulo envolve usualmente os segmentos da curva de demanda e da curva de oferta respectivamente e a terceira vértice ocorre na intervenção do governo pela incidência de impostos. Existe um ponto a partir da qual quanto maior a carga tributária menor a base de arrecadação pela retração da demanda e oferta pelo aumento dos preços (HARBERGER, 1959).

Tanzi e Zee (2000) explicam que em países em desenvolvimento, o estabelecimento de um sistema de arrecadação efetivo e eficiente encara alguns formidáveis desafios. O primeiro desses desafios é a estrutura da economia que dificulta a instituição e a coleta de certos impostos. O segundo é a limitada capacidade da administração de impostos. O terceiro é a escassez, baixa qualidade de informação básica.

Ainda conforme TANZI e ZEE (2000):

Países em desenvolvimento são comumente caracterizados por: uma grande participação da agricultura na geração de empregos, um grande mercado informal e empregos informais, um grande número de pequenos estabelecimentos, uma pequena 
participação dos salários no total nacional da renda; uma pequena parte dos gastos dos consumidores em grandes e modernos estabelecimentos e assim por diante.

Os sistemas de informação são então uma oportunidade para enfrentar os desafios apresentados. WALSH (2014) explica que em breve veremos a convergência tecnológica na fiscalização de impostos diretos e indiretos. Winn e Zhang (2013) também informam que a NF-e é uma tendência e os governos possuem os seguintes interesses nessa tecnologia:

- Sistemas eletrônicos confiáveis para geração e armazenamento de notas fiscais possuem menor custo para monitoramento da aderência ao pagamento de impostos;

- O largo uso de sistemas de informação capazes de gerar notas fiscais automaticamente incrementa a competitividade global de negócios locais.

A necessidade de um moderno sistema tributário tem sua importância também pela alta carga tributária de nosso país. A carga tributária per capita anual cresceu 277,3\% entre 2000 (quando era de R\$2.086,21) e 2013, quando chegou a R \$ 7.872,14. Em relação ao PIB, a carga tributária saiu de 30,03\% em 2000 para 36,27\% em 2012 (INSTITUTO ASSAF). Ainda, conforme o estudo do Instituto Assaf, os avanços dos sistemas de fiscalização da Receita Federal no combate à sonegação tiveram papel relevante no aumento da carga tributária.

No Brasil ainda existem poucos estudos sobre o assunto. Mattos et al (2013), publicaram um artigo analisando a influência da Nota Fiscal Eletrônica e do incentivo da Nota Fiscal Paulista na arrecadação do ICMS. Esse trabalho discute se o uso da NF-e no setor varejista atrelado a incentivos de premiação ao consumidor aumentava a arrecadação. Concluiu um aumento entre 5\% a 10\% da arrecadação em relação a outros estados do Brasil.

Segundo Confederação Nacional do Comércio de Bens, Serviços e Turismo, a Nota Fiscal Eletrônica (NF-e) é um documento digital (arquivo XML), emitido e armazenado de forma eletrônica, com o objetivo de documentar, para fins fiscais, uma operação de circulação de mercadorias ou uma prestação de serviços, ocorrida entre as partes. A validade jurídica da NF-e é garantida pela assinatura digital do remetente a consequente autorização de uso fornecida pelo fisco, antes da ocorrência do fato gerador. Já a Nota Fiscal Eletrônica de Serviços (NFS-e) é um documento de existência digital, gerado e armazenado eletronicamente em ambiente de TI Nacional pela Receita Federal, pela prefeitura, por outra entidade e/ou órgão público conveniado, para documentar as operações de prestação de serviços (DUARTE, 2009).

Esse trabalho diferencia-se da abordagem proposta por Mattos et al (2013) por identificar os impactos provocados pela implantação das notas fiscais eletrônicas de serviços, ao invés da análise da influência das notas fiscais de circulação de mercadorias.

\section{$2.2 \mathrm{O}$ certificado digital}

AZEVEDO e MARIANO (2014) explicam que com o SPED (Sistema Público de Escrituração Digital) e os seus projetos, passou a ser obrigatória a assinatura digital na ECD (Escrituração Contábil Digital), EFD (Escrituração Fiscal Digital) e na NF-e em cada documento fiscal ter a assinatura digital para assegurar a sua validade jurídica, é necessário que todos os contribuintes, independente do porte da empresa adquiram o certificado digital. A obrigação de uso do certificado ocorre também em todos os projetos do SPED desenvolvidos posteriormente.

A certificação digital é uma tecnologia que provê os mecanismos de segurança capazes de garantir autenticidade, confidencialidade e integridade às informações eletrônicas das mensagens e documentos trocados na Internet (PARISOTTO e FREIRE, 2011).

\subsection{Web services}

Segundo o World Wide Web Consortium um Web Service é um aplicativo de software identificado por um endereço de rede (URL), cujas interfaces e de ligação são capazes de serem definidos, descritos e descobertos por artefatos XML e suporta interações diretas com outras aplicações de software usando mensagens baseadas em XML através de protocolos com base na Internet.

A solução Web Services consiste na disponibilização de serviços na internet automatizados, disponibilizados 
pelos servidores utilizados pela da Administração Tributária Municipal. Essa solução tem como premissa a utilização de um browser ou aplicação no computador do cliente, que acessará, por meio da internet, os serviços do Web Service (Portal Prefeitura de Vassouras).

\section{4 A nota fiscal de serviços eletrônica}

O Projeto Nota Fiscal de Serviços Eletrônica (NFS-e) é uma iniciativa integrada da Receita Federal do Brasil (RFB) e da Associação Brasileira das Secretarias de Finanças das Capitais (Abrasf), buscando atendimento ao Protocolo de Cooperação ENAT n ${ }^{\circ}$ 02, de 7 de dezembro de 2007 (BEZERRA, 2010). Esse projeto possibilita a padronização e a melhoria da qualidade das informações, com redução de custos e eficácia, bem como o aumento da competitividade das empresas pela diminuição das obrigações acessórias e redução do custo-Brasil, em especial a dispensa da emissão e guarda de documentos em papel (BEZERRA, 2010). A Receita Federal do Brasil estuda vir a ser o repositório nacional das NFS-e, o que facilitaria ainda mais a adesão de pequenos municípios e a troca de informações entre os entes tributantes (Portal da Nfe).

Para a Nota Fiscal de Serviços Eletrônica (NFS-e) embora exista um protocolo de cooperação de criação de um modelo nacional único, os municípios possuem autonomia para definir e disponibilizar seu próprio sistema. Devido a isso, algumas cidades que implantaram a NFS-e no padrão da Abrasf, e outras seguiram modelos distintos (Confederação Nacional do Comércio de Bens, Serviços e Turismo). Em função das situações distintas, a única certeza existente é a responsabilidade das empresas prestadoras de serviços em cumprir a legislação tributária do seu município (Confederação Nacional do Comércio de Bens, Serviços e Turismo).

\begin{tabular}{ccc}
\hline & NF-e & NFS-e \\
\hline Armazenamento & Centralizado (Site Nota Fiscal & Descentralizado (Portais das Prefeituras) \\
Linguagem & Eletrônica) & XML \\
Assinatura & XML & Certificado Digital ou autenticação do \\
Objeto de & Certificado Digital & usuário \\
documentação & Circulação de mercadorias & Prestação de serviços \\
Consulta & Via Webservice & Via Webservice \\
Padrão de & Única & Diversificada \\
Arquivo & Via Webservice & Via Webservice ou manual \\
Envio & &
\end{tabular}

Quadro 1. Diferenças entre a NF-E e a NFS-e

Fonte: Elaborado pelos autores

\subsection{O ISSQN}

O Imposto sobre serviços de qualquer natureza (ISSQN ou apenas ISS), foi previsto na Emenda Constitucional n. 18, de $1^{\circ}-12-1965$. É um tributo que veio substituir o "Imposto sobre indústrias e profissões" (IIP), inicialmente estadual (a partir da Proclamação da República) e, após a Constituição Federal de 1946, um imposto municipal (TRISTÃO, 2003). O art. 156, III, da constituição federal de 1988 estabelece ser de competência dos municípios a instituição do ISS. Caberá aos Municípios, mediante a edição de uma lei ordinária, a instituição do ISS.

Assim, os mais de 5 mil municípios brasileiros editarão suas leis ordinárias municipais, instituidoras de ISS, em consonância com uma nacional lei complementar, que disciplinará, especialmente em relação aos impostos, as normas gerais definidoras dos fatos geradores, bases de cálculo e contribuintes (art. 146, III, “a”, CF) (SABBAG, 2013).

O ISS é um imposto municipal, dependente de lei ordinária, porém sua estrutura normativa está 
disciplinada em legislação complementar federal de âmbito nacional, a atual Lei Complementar n. 116/2003. O art. $3^{\circ}$, caput, da LC 116/2003 trouxe, assim, uma regra geral segundo a qual o sujeito ativo do ISS é o município do estabelecimento do prestador (ou, na falta deste, o do domicílio do prestador). Portanto, conforme TRISTÃO (2003), o "local da prestação do serviço" é o estabelecimento prestador, não importando onde viesse a ser prestado o serviço. Todavia, no próprio art. $3^{\circ}$, foram destacados 22 incisos (ou seja, 20 hipóteses, em face de dois vetos), admitindo, como exceção, o "local da prestação do serviço" como o município da prestação (SABBAG, 2013).

É sujeito passivo do ISS, segundo o art. $5^{\circ}$ da LC n. 116/2003, o prestador de serviço, na condição de empresa (v.g., hotel) ou de profissional autônomo (e.g., médico, dentista, contador, advogado, ou seja, todos os "vendedores dos bens imateriais"), com ou sem estabelecimento fixo. O fato gerador do ISS é a prestação - por empresa ou profissional autônomo, com ou sem estabelecimento fixo - de serviços constantes da Lista anexa à LC n. 116/2003, a qual enumera, aproximadamente, 230 serviços, divididos em 40 itens. Desse modo, em face do princípio da estrita legalidade tributária (art. 150, I, da CF; e art. 97 do CTN), os Municípios, para que possam cobrar o ISS dos prestadores de serviços, devem instituí-lo por meio de lei ordinária própria, que poderá adotar todos os itens da Lista de Serviços anexa à lei complementar, ou apenas alguns deles, sendo-lhes, porém, defeso criar serviços não previstos nessa norma complementar, sob pena de inconstitucionalidade (SABBAG, 2013).

\subsection{A Nfs-e na redução do custo da transação}

O surgimento da NFS-e possibilitou a disponibilização de empresas de contabilidade que oferecem seus serviços na internet. Isso ocorre porque as empresas de serviços possuem menos requisitos para a escrituração de suas transações. Dentre obrigações adicionais para empresas que circulam mercadorias, temos convênio ICMS 57/95 que orienta a emissão de documentos e escrituração de livros fiscais e a manutenção de informações em meio magnético, por contribuintes do IPI e/ou do ICMS usuários de sistema eletrônico de processamento de dados. Essa obrigatoriedade de armazenamento das informações em meio magnético impossibilita o armazenamento das informações em ambiente internet, o que não ocorre para empresas no setor de serviços.

Como um exemplo de empresa que fornece serviços de contabilidade na internet temos o site www. contabilizei.com.br que estabelece como valor inicial de seus serviços o valor de R\$49,00 mensal. Dentre os requisitos para ser cliente da Contabilizei a empresa não pode exercer atividades de comércio e indústria como também algumas atividades em prestação de serviços (Site Contabilizei). Como um comparativo, o Sindicato dos Escritórios de Contabilidade do Estado de Minas Gerais (Sinescontábil-MG) estabelece como preço de referência para 2015 um valor mínimo de R\$ 800,80 mensal (Site Sinescontábil-MG). Tendo como base os valores mínimos apresentados, um cliente usuário dos serviços da Contabilizei tem uma redução de custos anual de R \$ 9.021,60. Ao utilizar os serviços da empresa Contabilizei o cliente emite a nota fiscal diretamente a partir do site www. contabilizei.com.br e o cálculo dos impostos decorrentes do faturamento são disponibilizados on-line (Site Contabilizei).

Os sites das prefeituras que oferecem os serviços de nota fiscal eletrônica oferecem o armazenamento e a das notas fiscais eletrônicas de serviços emitidas, oferecendo uma alternativa de redução de custos inicial pela não obrigatoriedade na aquisição ou assinatura de uma mensalidade de software emissor de notas fiscais de terceiros. Os dados apresentados corroboram com os resultados apresentados por Winn e Zhang (2013) no potencial da nota fiscal eletrônica de aumento da competitividade global nos locais onde é implantado.

\section{MÉTODO}

O modelo utilizado para analisar o impacto da implantação da nota fiscal de serviços eletrônica nos municípios brasileiros foi o diferenças-em-diferenças, largamente utilizado a partir dos estudos de ASHENFELTER e CARD (1984). Será uma adaptação do modelo utilizado na pesquisa de Mattos et al (2013), de diferenças-emdiferenças na análise da implantação dos impactos do Programa Nota Fiscal Paulista para dois períodos.

O modelo diferenças-em-diferenças foi desenvolvido ASHENFELTER e CARD (1984) para estudos de experimentos naturais. Experimentos naturais ocorrem quando algum evento exógeno, como, por exemplo uma mudança política do governo, muda o ambiente no qual indivíduos, famílias, firmas ou cidades operam. Esse modelo é uma regressão múltipla a partir de uma variável dependente com variáveis dummy, variáveis de 
interpolação e variáveis de controle.

A amostra foi composta por dados relativos à arrecadação dos municípios brasileiros com mais de 100 mil habitantes para obter-se uma representação significativa dos impactos pós implantação da nota fiscal de serviços eletrônica no Brasil. Foram obtidos dados de 2007 a 2012 de arrecadação obtidas através de bases de dados públicas disponibilizadas pelos sites do IBGE e do Tesouro Nacional.

A coleta da informação do ano em que foi implantada a nota fiscal de serviços eletrônica em cada município se deu através de consultas nos sites das respectivas prefeituras em seus códigos tributários ou em notícias veiculadas a partir desses portais da obrigatoriedade do uso dessa ferramenta. O período a ser analisado corresponde ao início das primeiras implantações de notas fiscais eletrônicas de serviços no Brasil (2007) e segue até 2012, último ano com as variáveis desse estudo disponibilizadas nos sites anteriormente mencionados por município. Totalizam a amostra a ser utilizada um quantitativo de 300 municípios com população maior que 100 mil habitantes. Dessas, um quantitativo de 67 municípios foi excluído da base de dados pelo fato de não ter sido possível identificar o ano exato da implantação da nota fiscal de serviços eletrônica, restando 233 municípios na base.

Para a análise dos números levantados, verificou-se que o ano 2011 apresentou o maior número de implantações da nota fiscal de serviços eletrônica (52 municípios). Considerando a restrição de levantamento de dados fornecidos pelo Tesouro Nacional de dados de arrecadação até 2012, o ano de 2010 (terceiro ano com o maior número de implantações, com 39 municípios) possibilita também uma análise relevante por apresentar um período maior para a análise de dados após a implantação. Foram feitas então duas análises separadas considerando os anos de 2010 e 2011 como os de interesse a serem utilizados como parâmetros de pós implantação da nota fiscal de serviços eletrônica.

A importância dos anos de interesse deve-se ao fato de que as cidades que implantaram a nota fiscal de serviços eletrônica em um ano diferente devem ser excluídas da base já que a tendência de arrecadação nesses municípios estaria influenciada pela implantação dessa mudança em um ano diferente. Considerando como ano de interesse o ano de 2011 teremos como histórico de pós implantação o período abrangido por 2011 e 2012. Já para o ano de interesse 2010, teremos um histórico antes da implantação de 2007 a 2009 (3 anos) e um histórico de pósimplantação de 2010 a 2012 (3 anos).

$\mathrm{Na}$ análise com o ano de interesse 2011, as cidades que implantaram a NFs-e em 2007, 2008, 2009, 2010 e 2012 foram excluídas da base por já estarem sofrendo impactos da implantação em anos diferentes. Já na análise com o ano de interesse 2010, as cidades que implantaram a NFs-e em 2007, 2008, 2009, 2011 e 2012 foram excluídas da base por já estarem sofrendo impactos da implantação em anos diferentes.

As variáveis desse estudo serão os dados históricos de produto interno bruto per capita dos municípios, o produto interno bruto per capita do setor de serviços, a arrecadação de ISSQN per capita dos municípios, e as datas limite de implementação da nota fiscal de serviços eletrônica. O modelo básico utilizado nas estimativas é descrito por:

$\operatorname{ISSQNpC}=\beta_{0}+\beta_{1}{ }^{*} \operatorname{ImpANO}+\beta_{2} *$ PosANO $+\beta_{3}{ }^{*} \operatorname{ImpANOpos}+\beta_{4}{ }^{*} \mathrm{X}+\varepsilon$

Onde:

ISSQNpC $=$ arrecadação de ISSQN per capita no período.

$\mathrm{B}_{0}=$ intercepto de arrecadação de ISSQN per capita.

$\beta_{1}^{*}$ ImpANO = variável dummy com valores 1 para os municípios que implantaram a Nota Fiscal de Serviços Eletrônica no ano a ser analisado e 0 para os municípios que não implantaram a Nota Fiscal de Serviços no ano a ser analisado.

$\beta_{2}{ }^{*}$ PosANO = variável dummy com por valores 1 para ano maior ou igual ao ano a ser analisado e valores 0 para os anos menores que o ano a ser analisado.

$\beta_{3}{ }^{*} \operatorname{ImpANOpos}=$ variável de interação com os valores 1 para cidades que implantaram a nota fiscal de serviços eletrônica nos anos maiores ou iguais ao ano a ser analisado e valores 0 para cidades que não implantaram em anos anteriores ao ser analisado.

$\beta_{4}{ }^{*} \mathrm{X}=$ vetor de variáveis de controle a serem utilizadas, sendo elas:

$\mathrm{PIBpC}=$ PIB per capita

PIBSerpC $=$ PIB de serviços per capita,

$\varepsilon=$ variável de erro. 
Assim, no modelo o coeficiente $\beta_{3}$ na variável ImpANOpos mensura o efeito da interação a ser analisada para verificar se os municípios que implantaram a nota fiscal de serviços eletrônica no ano de interesse realmente tiveram um impacto positivo na arrecadação per capita de ISS. As variáveis de controle relacionadas ao PIB per capita, assumem que o volume da arrecadação de ISSQN per capita teria relação direta com o mesmo. A expectativa em termos de resultados é que quanto maior for o PIBpC (PIB per capita), maior seria a arrecadação de tributos em geral.

\subsection{Estatística descritiva e testes de médias}

Os municípios foram divididos em 2 grupos utilizando uma variável dummy que possui valores 1 para os municípios que implantaram a nota fiscal de serviços eletrônica e 0 para os municípios que não implantaram a nota fiscal de serviços eletrônica. Já os anos utilizados na análise foram divididos em 2 grupos utilizando uma variável dummy que possui valores 1 para os anos maiores ou iguais ao de interesse e 0 para valores menores que o de interesse. As análises foram realizadas duas vezes utilizando como parâmetros dos anos de interesse de implantação da nota fiscal de serviços eletrônica os anos 2010 e 2011.

Observa-se, com base nas Tabelas 1 e 3 que tanto os municípios que implantaram a NFs-e quanto os que não implantaram tiveram aumentos da arrecadação do PIB, PIB de serviços e ISSQN per capita semelhantes após 2010. Já a Tabela 2 apresenta o teste de médias que informa que os municípios que não implantaram a NFs-e apresentam uma curva crescente da arrecadação de ISSQN per capita. Essa curva crescente de arrecadação também foi confirmada nos municípios que implantaram a NFs-e pelo teste das médias apresentado na Tabela 4.

Tendo 2011 como ano de interesse, observam-se tanto para os municípios que não implantaram a NFs-e quanto para os municípios que implantaram resultados percentuais semelhantes (Tabelas 5 e 7). As Tabelas 6 e 8 confirmam que para esses 2 grupos também havia uma tendência do crescimento da arrecadação do ISSQN per capita.

Tabela 1. Estatística descritiva dos municípios que não implantaram a NFs-e

\begin{tabular}{|c|c|c|c|c|c|c|c|c|c|}
\hline \multirow[b]{2}{*}{ Variável } & \multicolumn{4}{|c|}{ Antes de 2010} & \multicolumn{4}{|c|}{ Após 2010} & \multirow[b]{2}{*}{$\begin{array}{l}\text { Var. \% } \\
\text { (B)/(A) }\end{array}$} \\
\hline & $\begin{array}{l}\text { Média } \\
\text { (A) }\end{array}$ & $\begin{array}{l}\text { Desvio } \\
\text { Padrão }\end{array}$ & Min. & Max. & $\begin{array}{c}\text { Média } \\
\text { (B) }\end{array}$ & $\begin{array}{l}\text { Desvio } \\
\text { Padrão }\end{array}$ & Min & Max & \\
\hline PIBpC & 19,38 & 16,30 & 3,68 & 101,47 & 25,88 & 20,90 & 5,07 & 108,09 & $33,52 \%$ \\
\hline PIBSerpC & 5,43 & 7,01 & 0,47 & 39,52 & 6,62 & 7,54 & 0,69 & 42,29 & $22,07 \%$ \\
\hline ISSQNpC & 0,11 & 0,09 & 0,00 & 0,52 & 0,17 & 0,16 & 0,02 & 0,92 & $61,80 \%$ \\
\hline \multicolumn{10}{|c|}{ Período analisado: 2007-2012. } \\
\hline \multicolumn{10}{|c|}{ Número de observações: 138 (antes e após 2010). } \\
\hline \multicolumn{10}{|c|}{ PIBpC - PIB per capita em R\$ mil. } \\
\hline \multicolumn{10}{|c|}{ PIBSerpC - PIB de serviços per capita em R\$ mil. } \\
\hline \multicolumn{10}{|c|}{ ISSQNpC - Impostos sobre serviços de qualquer natureza per capita em $\mathrm{R} \$$ mil. } \\
\hline
\end{tabular}

Fonte: Elaboração Própria

Tabela 2. Teste de médias do ISSQN per capita para os municípios que não implantaram a NFs-e

\begin{tabular}{lccccc}
\hline \multicolumn{1}{c}{ Grupo } & Média & Erro Padrão & Desvio Padrão & \multicolumn{2}{c}{ Intervalo de Confiança (95\%) } \\
\hline Antes 2010 & 0,1067 & 0,0079 & 0,0927 & 0,0911 & 0,1223 \\
Após 2010 & 0,1726 & 0,0137 & 0,1604 & 0,1456 & 0,1996 \\
Total & 0,1396 & 0,0081 & 0,1349 & 0,1236 & 0,1556 \\
Diferença & $-0,0659$ & 0,0158 & & $-0,0970$ & $-0,0349$
\end{tabular}

Período analisado: 2007-2012.

Número de Observações: 276.

$$
\text { Ha: diff }<0 \quad \text { Ha: diff } !=0 \quad \text { Ha: diff }>0
$$

$\operatorname{Pr}(\mathrm{T}<\mathrm{t})=0,0000$

$\operatorname{Pr}(|\mathrm{T}|>|\mathrm{t}|)=0,0000$

$\operatorname{Pr}(\mathrm{T}>\mathrm{t})=1,0000$

Fonte: Elaboração Própria 
Tabela 3. Estatística descritiva dos municípios que implantaram a NFs-e

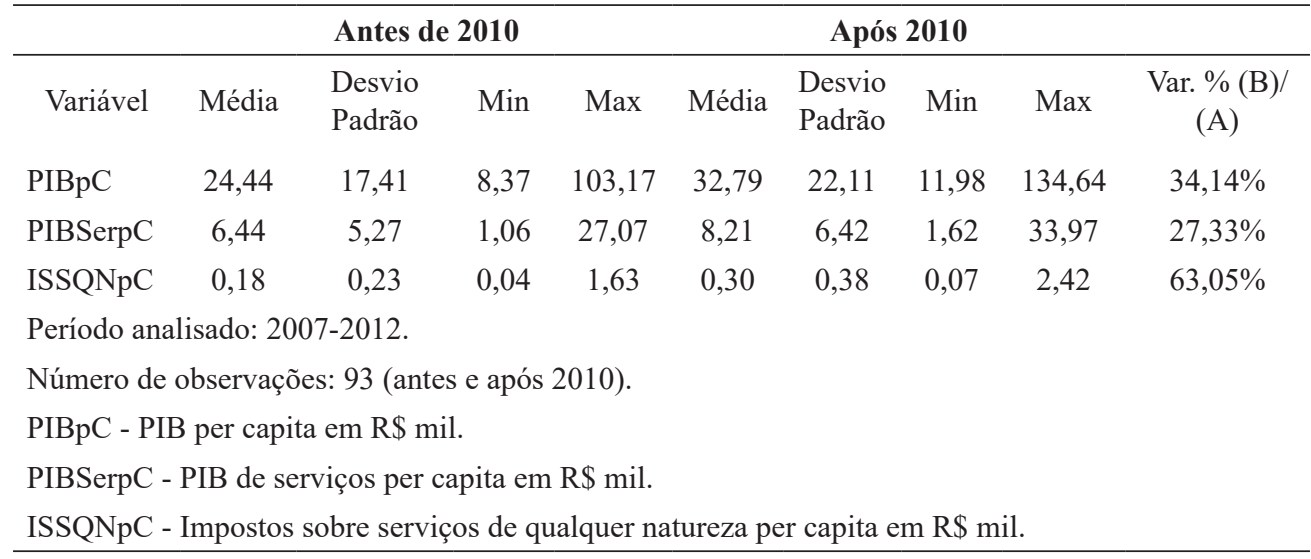

Fonte: Elaboração Própria

Tabela 4. Teste de médias do ISSQN per capita para os municípios que implantaram a NFs-e

\begin{tabular}{|c|c|c|c|c|c|}
\hline Grupo & Média & Erro Padrão & Desvio Padrão & \multicolumn{2}{|c|}{ Intervalo de Confiança (95\%) } \\
\hline Antes 2010 & 0,1818 & 0,0238 & 0,2293 & 0,1345 & 0,2290 \\
\hline Após 2010 & 0,2963 & 0,0393 & 0,3794 & 0,2182 & 0,3745 \\
\hline Total & 0,2391 & 0,0233 & 0,3178 & 0,1931 & 0,2850 \\
\hline Diferença & $-0,1146$ & 0,0460 & & $-0,2053$ & $-0,0239$ \\
\hline \multicolumn{6}{|c|}{ Período analisado: 2007-2012. } \\
\hline \multicolumn{6}{|c|}{ Número de Observações: 186.} \\
\hline \multicolumn{2}{|c|}{ Ha: $\operatorname{diff}<0$} & \multicolumn{2}{|c|}{ Ha: diff $!=0$} & \multicolumn{2}{|c|}{ Ha: $\operatorname{diff}>0$} \\
\hline \multicolumn{2}{|c|}{$\operatorname{Pr}(\mathrm{T}<\mathrm{t})=0,0068$} & \multicolumn{2}{|c|}{$\operatorname{Pr}(|\mathrm{T}|>|\mathrm{t}|)=0,0136$} & \multicolumn{2}{|c|}{$\operatorname{Pr}(\mathrm{T}>\mathrm{t})=0,9932$} \\
\hline
\end{tabular}

Fonte: Elaboração Própria

Tabela 5. Estatística descritiva dos municípios que não implantaram a NFs-e

\begin{tabular}{|c|c|c|c|c|c|c|c|c|c|}
\hline \multirow[b]{2}{*}{ Variável } & \multicolumn{4}{|c|}{ Antes de 2011} & \multicolumn{4}{|c|}{ Após 2011} & \multirow[b]{2}{*}{ Var. $\%(\mathrm{~B}) /(\mathrm{A})$} \\
\hline & $\begin{array}{c}\text { Média } \\
\text { (A) }\end{array}$ & $\begin{array}{c}\text { Desvio } \\
\text { adrão }\end{array}$ & Min. & Max. & $\begin{array}{c}\text { Média } \\
\text { (B) }\end{array}$ & $\begin{array}{l}\text { Desvio } \\
\text { Padrão }\end{array}$ & Min & $\operatorname{Max}$ & \\
\hline PIBpC & 20,57 & 17,32 & 3,68 & 105,44 & 26,75 & 21,46 & 5,24 & 108,09 & $30,08 \%$ \\
\hline PIBSerpC & 5,73 & 7,26 & 0,47 & 39,52 & 6,61 & 7,34 & 0,69 & 42,29 & $15,32 \%$ \\
\hline ISSQNpC & 0,12 & 0,11 & 0,00 & 0,81 & 0,18 & 0,17 & 0,03 & 0,92 & $58,07 \%$ \\
\hline \multicolumn{10}{|c|}{ Período analisado: 2007-2012. } \\
\hline \multicolumn{10}{|c|}{ Número de observações: 184 (antes 2011), 92 (após 2011). } \\
\hline \multicolumn{10}{|c|}{ PIBpC - PIB per capita em R \$ mil. } \\
\hline \multicolumn{10}{|c|}{ PIBSerpC - PIB de serviços per capita em $\mathrm{R} \$$ mil. } \\
\hline \multicolumn{10}{|c|}{ ISSQNpC - Impostos sobre serviços de qualquer natureza per capita em R\$ mil. } \\
\hline
\end{tabular}

Fonte: Elaboração Própria 
Tabela 6. Teste de médias do ISSQN per capita para os municípios que não implantaram a NFs-e

\begin{tabular}{lccccc}
\hline \multicolumn{1}{c}{ Grupo } & Média & Erro Padrão & Desvio Padrão & Intervalo de Confiança (95\%) \\
\hline Antes 2011 & 0,1170 & 0,0080 & 0,1088 & 0,1012 & 0,1328 \\
Após 2011 & 0,1849 & 0,0175 & 0,1675 & 0,1502 & 0,2196 \\
Total & 0,1396 & 0,0081 & 0,1349 & 0,1236 & 0,1556 \\
Diferença & $-0,0679$ & 0,0168 & & $-0,1009$ & $-0,0349$
\end{tabular}

Período analisado: 2007-2012.

Número de Observações: 184 (Antes 2011), 92 (após 2011).
Ha: diff $<0$
Ha: diff $!=0$
Ha: diff $>0$
$\operatorname{Pr}(\mathrm{T}<\mathrm{t})=0,0000$
$\operatorname{Pr}(|\mathrm{T}|>|\mathrm{t}|)=0,0001$
$\operatorname{Pr}(\mathrm{T}>\mathrm{t})=1,0000$

Fonte: Elaboração Própria

Tabela 7. Estatística descritiva dos municípios que implantaram a NFs-e

\begin{tabular}{|c|c|c|c|c|c|c|c|c|c|}
\hline \multirow[b]{2}{*}{ Variável } & \multicolumn{4}{|c|}{ Antes de 2011} & \multicolumn{4}{|c|}{ Após 2011} & \multirow[b]{2}{*}{ Var. $\%(\mathrm{~B}) /(\mathrm{A})$} \\
\hline & Média & $\begin{array}{l}\text { Desvio } \\
\text { Padrão }\end{array}$ & Min & Max & Média & $\begin{array}{l}\text { Desvio } \\
\text { Padrão }\end{array}$ & Min & Max & \\
\hline PIBpC & 18,84 & 9,02 & 4,24 & 55,64 & 24,50 & 9,62 & 6,76 & 47,70 & $30,04 \%$ \\
\hline PIBSerpC & 5,59 & 4,38 & 0,64 & 19,73 & 6,92 & 5,18 & 1,05 & 28,69 & $23,85 \%$ \\
\hline ISSQNpC & 0,12 & 0,13 & 0,00 & 1,03 & 0,18 & 0,17 & 0,04 & 1,22 & $51,60 \%$ \\
\hline \multicolumn{10}{|c|}{ Período analisado: 2007-2012. } \\
\hline \multicolumn{10}{|c|}{ Número de observações: 164 (antes 2011), 82 (após 2011). } \\
\hline \multicolumn{10}{|c|}{ PIBpC - PIB per capita em R\$ mil. } \\
\hline \multicolumn{10}{|c|}{ PIBSerpC - PIB de serviços per capita em R\$ mil. } \\
\hline \multicolumn{10}{|c|}{ ISSQNpC - Impostos sobre serviços de qualquer natureza per capita em R\$ mil. } \\
\hline
\end{tabular}

Tabela 8. Teste de médias do ISSQN per capita para os municípios que implantaram a NFs-e

\begin{tabular}{lccccc}
\hline \multicolumn{1}{c}{ Grupo } & Média & Erro Padrão & Desvio Padrão & \multicolumn{1}{c}{ Intervalo de Confiança (95\%) } \\
\hline Antes 2011 & 0,1210 & 0,0099 & 0,1262 & 0,1015 & 0,1404 \\
Após 2011 & 0,1834 & 0,0191 & 0,1729 & 0,1454 & 0,2214 \\
Total & 0,1418 & 0,0093 & 0,1462 & 0,1234 & 0,1601 \\
Diferença & $-0,0624$ & 0,0194 & $-0,1006$ & $-0,0242$ \\
Período analisado: 2007-2012. & & & \\
Número de Observações: 164 (Antes 2011), 82 (após 2011). & Ha: diff $>0$ \\
Ha: diff $<0$ & Ha: diff ! $=0$ & $\operatorname{Pr}(\mathrm{T}>\mathrm{t})=0,9993$ \\
$\operatorname{Pr}(\mathrm{T}<\mathrm{t})=0,0007$ & $\operatorname{Pr}(|\mathrm{T}|>|\mathrm{t}|)=0,0015$ &
\end{tabular}

Fonte: Elaboração Própria

\subsection{Teste de robustez: região metropolitana do Rio de Janeiro}

A Constituição Federal de 1988 deixa a cargo dos estados a instituição de Regiões Metropolitanas que seriam constituídas por agrupamentos de municípios limítrofes, para integrar a organização, o planejamento e a execução de funções públicas de interesse comum. Conforme o Instituto Brasileiro de Geografia e Estatística (IBGE), as regiões metropolitanas são áreas compostas por um conjunto de cidades contíguas e com integração socioeconômica a um município de grande porte. As características comuns mencionadas tornam-se um critério para a realização de um teste adicional para observar os impactos pela implantação da nota fiscal de serviços eletrônica em municípios de características semelhantes.

O Portal do IBGE lista 39 regiões metropolitanas hoje no Brasil. A partir da amostra composta por dados relativos à arrecadação dos municípios brasileiros com mais de 100 mil habitantes foram agrupadas as regiões 
metropolitanas. A Região Metropolitana do Rio de Janeiro mostrou-se a região com a maior padronização do ano onde houve a implantação da nota fiscal de serviços eletrônica, fornecendo a maior amostra de municípios para a realização de um teste adicional.

As Tabelas 9 e 10 apresentam as variações percentuais para os municípios que não implantaram a NFs-e em 2010 e as variações para os municípios que implantaram a NFs-e respectivamente. Observa-se nesse teste de robustez que os municípios que não implantaram a NFs-e apresentam incrementos ligeiramente maiores que os municípios que implantaram. Tendo como parâmetro de ano de interesse o 2011 os resultados invertem-se, apresentando um resultado superior após 2011 para os municípios que implantaram a NFs-e em comparação aos municípios que não implantaram.

Tabela 9. Estatística descritiva dos municípios que não implantaram a NFs-e na Região metropolitana do Rio de Janeiro

\begin{tabular}{|c|c|c|c|c|c|c|c|c|c|}
\hline \multirow[b]{2}{*}{ Variável } & \multicolumn{4}{|c|}{ Antes de 2010} & \multicolumn{4}{|c|}{ Após 2010} & \multirow[b]{2}{*}{ Var. \% (B)/(A) } \\
\hline & Média & $\begin{array}{l}\text { Desvio } \\
\text { Padrão }\end{array}$ & Min. & Max. & Média & $\begin{array}{l}\text { Desvio } \\
\text { Padrão }\end{array}$ & Min. & Max. & \\
\hline PIBpC & 7,61 & 0,92 & 6,47 & 9,34 & 10,99 & 2,09 & 9,13 & 15,89 & $44,39 \%$ \\
\hline PIBSerpC & 1,02 & 0,40 & 0,67 & 1,73 & 1,86 & 1,39 & 1,09 & 5,39 & $81,30 \%$ \\
\hline ISSQNpC & 0,03 & 0,01 & 0,02 & 0,04 & 0,05 & 0,01 & 0,03 & 0,07 & $89,49 \%$ \\
\hline \multicolumn{10}{|c|}{ Período analisado: 2007-2012. } \\
\hline \multicolumn{10}{|c|}{ Número de observações: 9 (antes e após 2010). } \\
\hline \multicolumn{10}{|c|}{ PIBpC - PIB per capita em R\$ mil. } \\
\hline \multicolumn{10}{|c|}{ PIBSerpC - PIB de serviços per capita em R $\$$ mil. } \\
\hline ISSQNpC - & ostos sob & serviços & qualquer & tureza $p$ & capita em & $\mathrm{R} \$$ mil. & & & \\
\hline
\end{tabular}

Fonte: Elaboração Própria

Tabela 10. Estatística descritiva dos municípios que implantaram a NFs-e na região Metropolitana do Rio de Janeiro

\begin{tabular}{|c|c|c|c|c|c|c|c|c|c|}
\hline \multirow[b]{2}{*}{ Variável } & \multicolumn{4}{|c|}{ Antes de 2010} & \multicolumn{4}{|c|}{ Após 2010} & \multirow[b]{2}{*}{ Var. $\%(B) /(A)$} \\
\hline & Média & $\begin{array}{l}\text { Desvio } \\
\text { Padrão }\end{array}$ & Min. & Max. & Média & $\begin{array}{l}\text { Desvio } \\
\text { Padrão }\end{array}$ & Min. & Max. & \\
\hline PIBpC & 18,16 & 7,14 & 8,37 & 27,56 & 24,36 & 9,29 & 11,98 & 34,57 & $34,15 \%$ \\
\hline PIBSerpC & 2,17 & 0,86 & 1,06 & 3,61 & 3,59 & 1,90 & 1,62 & 7,26 & $65,07 \%$ \\
\hline ISSQNpC & 0,22 & 0,15 & 0,04 & 0,45 & 0,34 & 0,23 & 0,07 & 0,67 & $52,42 \%$ \\
\hline \multicolumn{10}{|c|}{ Período analisado: 2007-2012. } \\
\hline \multicolumn{10}{|c|}{ Número de observações: 11 (antes e após 2010). } \\
\hline \multicolumn{10}{|c|}{ PIBpC - PIB per capita em R\$ mil. } \\
\hline \multicolumn{10}{|c|}{ PIBSerpC - PIB de serviços per capita em R \$ mil. } \\
\hline \multicolumn{10}{|c|}{ ISSQNpC - Impostos sobre serviços de qualquer natureza per capita em $\mathrm{R} \$$ mil. } \\
\hline
\end{tabular}


Tabela 11. Estatística descritiva dos municípios que não implantaram a NFs-e na região Metropolitana do Rio de Janeiro

\begin{tabular}{|c|c|c|c|c|c|c|c|c|c|}
\hline \multirow[b]{2}{*}{ Variável } & \multicolumn{4}{|c|}{ Antes de 2011} & \multicolumn{4}{|c|}{ Após 2011} & \multirow[b]{2}{*}{ Var. \% (B)/(A) } \\
\hline & Média & $\begin{array}{l}\text { Desvio } \\
\text { Padrão }\end{array}$ & Min. & Max. & Média & $\begin{array}{l}\text { Desvio } \\
\text { Padrão }\end{array}$ & Min. & Max. & \\
\hline PIBpC & 8,16 & 1,31 & 6,47 & 10,71 & 11,59 & 2,34 & 9,48 & 15,89 & $42,01 \%$ \\
\hline PIBSerpC & 1,14 & 0,47 & 0,67 & 2,12 & 2,04 & 1,69 & 1,09 & 5,39 & $79,35 \%$ \\
\hline ISSQNpC & 0,03 & 0,01 & 0,02 & 0,06 & 0,06 & 0,01 & 0,04 & 0,07 & $74,85 \%$ \\
\hline \multicolumn{10}{|c|}{ Período analisado: 2007-2012. } \\
\hline \multicolumn{10}{|c|}{ Número de observações: 12 (antes 2011), 6 (após 2011). } \\
\hline \multicolumn{10}{|c|}{ PIBpC - PIB per capita em R\$ mil. } \\
\hline \multicolumn{10}{|c|}{ PIBSerpC - PIB de serviços per capita em R $\$$ mil. } \\
\hline \multicolumn{10}{|c|}{ ISSQNpC - Impostos sobre serviços de qualquer natureza per capita em R\$ mil. } \\
\hline
\end{tabular}

Fonte: Elaboração Própria

Tabela 12. Estatística descritiva dos municípios que implantaram a NFs-e na região metropolitana do Rio de Janeiro

\begin{tabular}{|c|c|c|c|c|c|c|c|c|c|}
\hline \multirow[b]{2}{*}{ Variável } & \multicolumn{4}{|c|}{ Antes de 2011} & \multicolumn{4}{|c|}{ Após 2011} & \multirow[b]{2}{*}{ Var. $\%(B) /(A)$} \\
\hline & Média & $\begin{array}{l}\text { Desvio } \\
\text { Padrão }\end{array}$ & Min. & Max. & Média & $\begin{array}{l}\text { Desvio } \\
\text { Padrão }\end{array}$ & Min. & Max. & \\
\hline PIBpC & 8,88 & 1,98 & 6,81 & 12,88 & 18,84 & 10,52 & 12,41 & 39,47 & $112,17 \%$ \\
\hline PIBSerpC & 1,43 & 1,08 & 0,64 & 3,91 & 8,25 & 10,58 & 1,17 & 28,69 & $477,12 \%$ \\
\hline ISSQNpC & 0,04 & 0,01 & 0,02 & 0,06 & 0,08 & 0,03 & 0,05 & 0,13 & $134,81 \%$ \\
\hline \multicolumn{10}{|c|}{ Período analisado: 2007-2012. } \\
\hline \multicolumn{10}{|c|}{ Número de observações: 12 (antes 2011), 6 (após 2011). } \\
\hline \multicolumn{10}{|c|}{ PIBpC - PIB per capita em R\$ mil. } \\
\hline \multicolumn{10}{|c|}{ PIBSerpC - PIB de serviços per capita em R $\$$ mil. } \\
\hline ISSQNpC - & postos sob & e serviços & qualqu & natureza & capita e & $\mathrm{R} \$$ mil. & & & \\
\hline
\end{tabular}

Fonte: Elaboração Própria

\section{ANÁLISE DOS RESULTADOS}

\subsection{Resultados obtidos com os dados coletados}

Nessa sessão serão apresentados os resultados das regressões múltiplas na aplicação do modelo diferenças em diferenças com a utilização de dados em painel, analisando a variável dependente ISSQN per capita, onde chegou-se aos dados constantes nas Tabelas 13 e 14. É possível verificar pela variável de interação Imp2010pos (Tabela 13 - ano de interesse 2010) e pela variável de interação Imp2011pos (Tabela 14 - ano de interesse 2011) um efeito positivo causado pela implantação da nota fiscal de serviços eletrônica. Entretanto, considerando tanto para o ano de interesse 2010 como o ano de interesse 2011 a análise de $\mathrm{P}$ valor esse efeito não se mostrou significativo.

Em adição aos percentuais de evolução semelhantes entre os municípios que não implantaram a NFs-e e os municípios que implantaram apresentados na estatística descritiva é possível concluir que não houveram evidências fortes de que a implantação da nota fiscal de serviços eletrônica causou impactos positivos na arrecadação do ISSQN per capita. No que se refere ao PIB per capita, se confirmou a hipótese de que aquelas cidades com maior PIB per capita, tendem a possuir uma maior arrecadação de ISSQN em termos per capita. Entretanto no que toca ao PIB serviços per capita, um maior valor deste não implicou necessariamente numa maior arrecadação per capita de ISSQN, esboçando que a disponibilidade de PIB de serviços per capita maior, não necessariamente implica em maior arrecadação do ISSQN per capita na amostra de cidades em estudo, que correspondem aquelas mais 
populosas. Isso implica que essas cidades podem ter sua arrecadação mais distribuída por outras fontes tributárias.

Tabela 13. Diff-in-Diff ISSQNpC (Ano Base 2010)

\begin{tabular}{lcccccc}
\hline \multicolumn{1}{c}{ ISSQNpC } & Coef. & Erro Padrão & T & \multicolumn{2}{c}{ P Valor } & Intervalo de Confiança (95\%) \\
\hline Pos2010 & 0,0022 & 0,0143 & 0,1500 & 0,8780 & $-0,0259$ & 0,0302 \\
Imp2010 & 0,0268 & 0,0142 & 1,8900 & 0,0590 & $-0,0011$ & 0,0546 \\
Imp2010Pos & 0,0340 & 0,0264 & 1,2900 & 0,1980 & $-0,0179$ & 0,0859 \\
PIBpC & 0,0127 & 0,0021 & 6,1100 & 0,0000 & 0,0086 & 0,0168 \\
PIBSerpC & $-0,0156$ & 0,0040 & $-3,9300$ & 0,0000 & $-0,0234$ & $-0,0078$ \\
cons & $-0,0545$ & 0,0212 & $-2,5700$ & 0,0100 & $-0,0962$ & $-0,0128$ \\
Número de Observações: 462. & & & & & \\
F(5, 456)=24,80 & & & & & & \\
Prob $>$ F $=0,0000$ & & & & & & \\
R-quadrado $=0,6732$ & & & & & & \\
\hline
\end{tabular}

Fonte: Elaboração Própria

Tabela 14. Diff-in-Diff ISSQNpC (Ano Base 2011)

\begin{tabular}{lcccccc}
\hline ISSQNpC & Coef. & Erro Padrão & T & P Valor & Intervalo de Confiança (95\%) \\
\hline Pos2011 & 0,0290 & 0,0109 & 2,6700 & 0,0080 & 0,0076 & 0,0503 \\
Imp2011 & 0,0152 & 0,0103 & 1,4700 & 0,1410 & $-0,0051$ & 0,0355 \\
Imp2011Pos & $-0,0004$ & 0,0226 & $-0,0200$ & 0,9860 & $-0,0448$ & 0,0440 \\
PIBpC & 0,0068 & 0,0011 & 6,4000 & 0,0000 & 0,0047 & 0,0089 \\
PIBSerpC & $-0,0034$ & 0,0021 & $-1,6200$ & 0,1050 & $-0,0075$ & 0,0007 \\
cons & $-0,0029$ & 0,0115 & $-0,2500$ & 0,8030 & $-0,0254$ & 0,0196 \\
Número de Observações: 522. & & & & & \\
F(5, 516)=28,25 & & & & & & \\
Prob > F = 0,0000 & & & & & & \\
R-quadrado = 0,4271 & & & & & & \\
\hline
\end{tabular}

Fonte: Elaboração Própria

\subsection{Resultados na região metropolitana do Rio de Janeiro}

Nos resultados das regressões múltiplas na aplicação do modelo diferenças em diferenças com a utilização de dados em painel da região Metropolitana do Rio de Janeiro temos novamente uma influência positiva da influência da implantação da NFS-e na arrecadação do ISSQN per capita. Esse resultado, contudo, também não pôde ser considerado significativo pelos $\mathrm{P}$ valores apresentados.

O teste de robustez teve como propósito analisar comparativamente municípios dentro de uma mesma região metropolitana. Eventualmente, por força da adoção da NFS-e, poderiam ter sido adotadas intensivamente estratégias de migração de empresas para jurisdições que não tivessem implementados as NFS-e, com receio de um eventual aumento da fiscalização, entretanto os resultados não evidenciaram que esse presumível fenômeno de migração de jurisdições, tivesse efeito nos resultados.

\section{CONCLUSÕES}

Este trabalho teve tema central a análise da evolução dos sistemas informacionais, em especial a implantação de um modelo de notas fiscais eletrônicas, que vem sendo pelos governos municipais como uma forma de possibilitar uma maior arrecadação para as administrações tributárias. 
No Brasil, houve uma evolução considerável a partir da execução de um plano de trabalho plurianual iniciado a partir do $1^{\circ}$ Encontro Nacional das Administrações Tributárias, ocorrido em 2004.

Este trabalho mostrou, entretanto, que dentre as variáveis do modelo, a implantação de um sistema de notas fiscais eletrônicas (no caso estudado, o de serviços - NFS-e) apresentou em uma análise agregada tanto para os municípios que não implantaram quanto para os municípios que implantaram a NFS-e curvas de crescimento percentuais semelhantes no PIB, PIB de serviços e ISSQN per capita (considerando como anos de implantação o 2010 e 2011 respectivamente). Na regressão múltipla de diferenças em diferenças utilizada para analisar o impacto da nota fiscal de serviços eletrônica os impactos positivos apresentados pela implantação dessa ferramenta não puderam ser considerados significativos devido aos $\mathrm{P}$ valores apresentados.

No teste de robustez para a região metropolitana do Rio de Janeiro verificou-se resultados melhores para os municípios que não implantaram a NFs-e considerando como ano de interesse o 2010 e resultados melhores para municípios que implantaram a NFs-e considerando como ano de interesse o 2011. Na análise da regressão múltiplas o impacto da implantação da NFs-e não apresentaram P valores que pudessem ser considerados significativos.

Sendo assim, pôde-se inferir do estudo que a implantação da nota fiscal de serviços eletrônica não impactou no aumento da arrecadação do ISSQN per capita nos municípios apresentados. Esse resultado, de certo modo, era previsível, pois a mudança de método da nota convencional para eletrônica, não implicaria necessariamente numa redução eventual dos níveis de sonegação (ou informalidade), ou num necessário estimulo ao compliance tributário por parte do sujeito passivo das obrigações tributária. O resultado esperado com a nova metodologia seria efetivamente o aprimoramento da Administração Tributária (sujeito ativo), com processos mais céleres para arrecadação e cobrança de ISSQN, num universo crescente de operações que não poderiam ser controladas pelos métodos tradicionais.

Inobstante os resultados apresentados, não se podem deixar de reconhecer que como todo trabalho empírico estamos sujeitos a eventuais limitações. Entre todas destacam-se o fato de que a amostra ficou concentrado naqueles municípios mais populosos, talvez os resultados podem ser outros se ampliássemos a análise para um universo maior de município. Peculiaridades dos municípios mais populosos podem explicar uma diferença de tratamento em relação aos demais municípios menos populosos que são a imensa maioria.

Registre-se, que os resultados desta pesquisa orientada para municípios foram semelhantes aos apresentados nos estudos de Mattos et al (2013) que analisou o impacto do Programa Nota Fiscal Paulista sobre a arrecadação do Estado de São Paulo, onde o impacto desse programa foi igualmente identificado como limitado para fins de aumento da receita tributária estadual.

Como agenda de futuras pesquisas, sugere-se aprofundar a análise das eventuais economias em termos de custo de transação da adoções da NFS-e, pois, é nesse ponto que certamente se identifica o potencial de benefício sociais. Outro estudo relevante seria verificar os efeitos qualitativos em termos de melhoria de serviços oferecidos pela Administração Tributária com a adoção da NFS-e. Em suma, inegavelmente, trata-se de uma área promissora para pesquisas futuras, ainda muito carente de estudos no Brasil.

\section{REFERÊNCIAS}

ASHENFELTER, Orley; CARD, David. Using the longitudinal structure of earnings to estimate the effect of training programs. National Bureau of Economic Research, 1984.

ASSOCIAÇÃO BRASILEIRA DAS SECRETARIAS DE FINANÇAS DAS CAPITAIS (ABRASF). Disponível em: <http://www.abrasf.org.br/arquivos/files/NFSE-NACIONAL Modelo Conceitual\%20versao\%202-02. pdf $>$. Acesso em 02 jul. 2015.

AZEVEDO, Osmar Reis; MARIANO, Paulo Antônio. SPED Sistema Público de Escrituração Digital. 6. Ed. São Paulo: IOB, 2014.

BEZERRA, Marcelo Pinheiro. OS IMPACTOS SOBRE O PROFISSIONAL DE CONTABILIDADE COM IMPLANTANÇÃO DO SISTEMA PÚBLICO DE ESCRITURAÇÃO DIGITAL (SPED). Curso de graduação em Ciências Contábeis da Faculdade Lourenço Filho, 2010.

BRASIL. Constituição (1988). Constituição da República Federativa do Brasil. Brasília, DF, Carta Magna Senado Federal, 1998.

Confederação Nacional do Comércio de Bens, Serviços e Turismo. Disponível em: < http://www.cnc.org.br/ sites/default/files/arquivos/sped-2aedicao.pdf> . Acesso em 02 abr. 2015.

Contabilizei - Contabilidade on-line. Disponível em: <www.contabilizei.com.br $>$. Acesso em 20 ago. 2015. 
DUARTE, Roberto Dias. Big brother fiscal III: o Brasil na era do conhecimento: como a certificação digital, SPED e NF-e estão transformando a Gestão Empresarial no Brasil. São Paulo: Ideas@ work, p. 73, 2009.

HARBERGER, Arnold C.. Monopoly and resource allocation. American Economic Review no.2, 1954, V. 44, p.77-87.

. Using the resources at hand more effectively. American Economic Review no.2, 1959, V. 49, p.134-146.

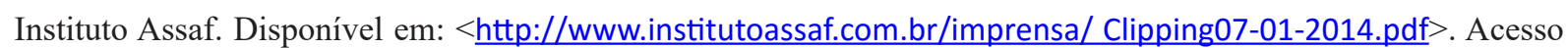
em 28 jan. 2014.

MATTOS, Enlinson; ROCHA, Fabiana; TOPORCOV, Patricia. Programas de incentivos fiscais são eficazes?: evidência a partir da avaliação do impacto do programa nota fiscal paulista sobre a arrecadação de ICMS. Revista Brasileira de Economia, v. 67, n. 1, p. 97-120, 2013.

MELO, PAULO ROBERTO DE SOUSA; MÖLLER JR, OSCAR. Panorama da Automação Comercial no Brasil. BNDES SETORIAL, v. 5, p. 129, 1997.

OLIVEIRA, José Teófilo. Reforma tributária: uma resenha. Coletânea de artigos. Vitória: 2002.

PARISOTTO, Cristiano; FREIRE, Anésio. NOTA FISCAL ELETRÔNICA-DESENVOLVENDO E IMPLANTANDO UM MÓDULO EMISSOR NF-E. Revista Ceciliana: Ago. 2010.

Portal da Nota Fiscal Eletrônica. Perguntas Frequentes. Disponível em: < http://www.nfe.fazenda.gov.br/portal/ perguntasFrequentes.aspx>. Acesso em 28 jan. 2014.

Portal Instituto Brasileiro de Geografia e Estatística. Disponível em: < http://www.ibge.gov.br/home/estatistica/ populacao/censo2010/sinopse/sinopse_tab_rm_zip.shtm>. Acesso em 20 ago 2015.

Portal Nfe do Brasil. Disponível em: < http://www.nfedobrasil.com.br/2010/12/duvidas-com-a-nota-fiscal-deservicos/>. Acesso em 17 jul. 2015.

Portal Prefeitura de Vassouras. Disponível em: < http://vassouras.rj.gov.br/arquivos/ NFSe_ModeloConceitual. pdf $>$. Acesso em 01 set. 2015.

QUEST, M., \& SHARMA, B. THE TECHNOLOGY OF TAX. Accountancy Age, 20. 2009.

Protocolo de Cooperação No 2/2007 - IV ENAT. Disponível em: < http://www.receita.fazenda.gov.br/Legislacao/ RecomendacoesCGSN/Protocolos/2007/protocolo2.htm>. Acesso em 17 jul 2015.

Receita da Fazenda. Disponível em: < http://www1.receita.fazenda.gov.br/ sistemas/nfse/o-que-e.htm>. Acesso em 15 jan 2015.

SABBAG, Eduardo de Moraes. Manual de direito tributário. 5. Ed. Saraiva, 2013.

Secretaria de Estado da Fazenda do Espírito Santo. Disponível em: < http://internet.sefaz.es.gov.br/informacoes/ nfe/historico.php>. Acesso em 29 jan. 2014.

Sindicato dos Escritórios de Contabilidade Auditoria e Perícias Contábeis no Estado de Minas Gerais - Sinescontábil - MG. Disponível em: <http://www.sinescontabil.com.br/>. Acesso em 30 jul. 2015.

TANZI, Vito; ZEE, Howell H. Tax policy for emerging markets: developing countries. National tax journal, p. 299-322, 2000.

TRISTÃO, José Américo Martelli. A Administração Tributária dos Municípios Brasileiros: Uma avaliação do desempenho da arrecadação. 2003. Tese de Doutorado.

WALSH, Chris. "The future of transaction tax management and technology." International Tax Review, p. 23, 2012.

WINN, Jane; ZHANG, Angela. China’s Golden Tax Project: A Technological Strategy for Reducing VAT Fraud. Social Science Research Network Working Paper Series, 2010.

WOOLDRIDGE, Jeffrey M. Introductory econometrics: A modern approach, 2e. Thomson South, 2003. 